\title{
Schulische Medienkompetenzförderung in einer digitalen Welt: Über welche digitalen Kompetenzen verfügen angehende Lehrkräfte?
}

\author{
Martin Senkbeil, Jan Marten Ihme, Christian Schöber \\ Leibniz-Institut für die Pädagogik der Naturwissenschaften und Mathematik an der Universität Kiel
}

Zusammenfassung: Die fortschreitende Digitalisierung der Gesellschaft stellt Schulen vor die Herausforderung, Kindern und Jugendlichen stärker als bisher digitale Kompetenzen zu vermitteln. Angehende wie auch aktive Lehrpersonen nehmen hierbei eine Schlüsselrolle ein, da sie sowohl über medienpädagogische als auch über digitale Kompetenzen verfügen müssen, um die Lehr- und LernPotenziale digitaler Medien systematisch ausschöpfen zu können. Obwohl es an empirischen Studien zu digitalen Kompetenzen von Lehramtsstudierenden mangelt, werden ihnen häufig defizitäre und im Sinne einer Negativselektion geringere digitale Kompetenzen unterstellt als Studierenden anderer Fachrichtungen. Dieser offenen Frage gehen wir anhand von Sekundäranalysen des Nationalen Bildungspanels (NEPS) nach, indem die Daten von $N=1518$ angehenden Studierenden sowie von $N=1766$ fortgeschrittenen Studierenden im sechsten Fachsemester analysiert wurden. Die Analysen weisen sowohl für angehende als auch für fortgeschrittene Lehramtsstudierende auf Kompetenzdefizite im Vergleich zu Studierenden anderer Fachrichtungen hin, die einem kleinen Effekt entsprechen $(0.32 \leq d \leq 0.47)$. Im Sinne einer negativen Binnenselektion verfügen vor allem Lehramtsstudierende ohne mathematisch-naturwissenschaftliches Unterrichtsfach über vergleichsweise geringe digitale Kompetenzen. Weiterführend kann in Bezug auf normativ festgelegte Mindeststandards digitaler Kompetenzen gezeigt werden, dass diese von substanziellen Anteilen der angehenden und fortgeschrittenen Lehramtsstudierenden (25\% bzw. 53\%) nicht erreicht werden. Abschließend diskutieren wir mögliche Implikationen für die Lehramtsausbildung.

Schlüsselbegriffe: Digitale Kompetenz, Lehramtsstudium, MINT-Fächer, Mindeststandards

\section{Dissemination of media literacy at school in a digital world: Are teacher candidates digitally competent?}

Summary: The progressive digitalization of society presents schools with the challenge of conveying digital skills to children and young people to a greater extent than before. Prospective as well as active teachers play a key role, as they must have both pedagogical media competencies and digital literacy skills to systematically utilize the teaching and learning potential of digital media. Although there is a lack of empirical studies on teacher candidates' digital literacy, these candidates are often assumed to have deficient and lower digital skills than students in other subject areas due to negative selection. We examined this question by analyzing data from the German National Education al Panel Study (NEPS) provided by $N=1518$ first-year students and $N=1766$ advanced students in the sixth semester. The results showed that first-year teacher candidates as well as teacher candidates in the sixth semester had lower digital literacy skills than students in other subject areas. The differences correspond to small effect sizes $(0.32 \leq d \leq 0.47)$. Closer inspection of the teacher candidates by study majors revealed that students without mathematics or any field of science as subjects had particularly low digital literacy skills, indicating a negative self-selection of this group. The results also show that a substantial number of first-year teacher candidates $(25 \%)$ as well as teacher candidates in the sixth semester $(53 \%)$ did not meet the minimum level of proficiency in digital skills as defined by an expert panel. Finally, we discuss possible implications for teacher training.

Keywords: Digital literacy, skills, teacher candidates, STEM, minimum level of proficiency in digital literacy skills 
Die fortschreitende Digitalisierung der Gesellschaft stellt Schulen weltweit vor neue Herausforderungen. Die Aufgabe, auf eine selbstbestimmte Teilhabe in der Gesellschaft vorzubereiten, beinhaltet mehr denn je die schulische Vermittlung von digitaler Medienkompetenz. Bei dieser Herausforderung nehmen die Lehrpersonen eine Schlüsselrolle ein. Diese müssen über medienpädagogische Kompetenzen verfügen, um die Potenziale digitaler Medien beim Lehren und Lernen systematisch ausschöpfen zu können (Herzig \& Martin, 2018; Kammerl \& Mayrberger, 2011). Eine grundlegende Voraussetzung für erfolgreiches medienpädagogisches Handeln stellt der eigene kompetente Umgang mit Informations- und Kommunikationstechnologien (Information and Communication Technologies, ICT Literacy) dar (Hatlevik, 2017; Herzig \& Martin, 2017; Tulodziecki, 2012).

Vielfach wird vermutet, dass substanzielle Anteile von angehenden und aktiven Lehrkräften in Deutschland für diese Aufgabe nicht über eine ausreichende ICT Literacy verfügen (z. B. Herzig \& Grafe, 2007; Lorenz, Gerick, Wendt \& Weischenberg, 2016; Zylka \& Müller, 2011). Dabei wird angenommen, dass Personen mit vergleichsweise distanzierten Haltungen zu digitalen Medien das Lehramtsstudium wählen. Das heißt, dass es bereits durch die Berufswahl zu einer Negativselektion im Sinne einer spezifischen Selbstselektion der Studierenden kommt und Lehramtsstudierende über geringere digitale Kompetenzen verfügen als Studierende anderer Fachrichtungen (Kammerl \& Pannarale, 2007). Gleichwohl liegt für diese Annahme bislang keine belastbare empirische Evidenz vor: Mit Ausnahme weniger Studien an nichtrepräsentativen Gelegenheitsstichproben (Herzig, Martin, Schaper \& Ossenschmidt, 2015; Zylka \& Müller, 2011) gibt es keine Befunde zu digitalen Kompetenzen angehender und aktiver Lehrkräfte, die auf objektiven Tests beruhen. Die weitaus meisten Arbeiten, die auf vergleichsweise geringe digitale Kompetenzen von Lehrkräften hinweisen, basieren auf Selbsteinschätzungen (z. B. Lorenz et al. 2016; Pettersson, 2018), die jedoch keine validen Aussagen über ihre tatsächlichen Kompetenzen erlauben (Kuhlemeier $\&$ Hemker, 2007).

Im vorliegenden Beitrag wird daher die ICT Literacy von angehenden (Schülerinnen und Schüler der 12. Klasse, die direkt nach dem Abitur ein Lehramtsstudium aufgenommen haben) und fortgeschrittenen Lehramtsstudierenden (sechstes Fachsemester) anhand von Sekundäranalysen des Nationalen Bildungspanels (National Educational Panel Study, NEPS) untersucht. Zum einen wird die ICT Literacy beider Lehramtsstudierenden-Gruppen mit denen von Studierenden anderer Fachrichtungen verglichen. Im Sinne einer negativen Binnenselektion (z. B. Klusmann, Trautwein, Lüdtke, Kunter \& Baumert, 2009) wird weitergehend geprüft, ob sich innerhalb der Lehramtsstudierenden Kompetenzunterschiede in Abhängigkeit von der gewählten Fächerkombination finden lassen. Darüber hinaus können die erzielten Kompetenzwerte in kriterial verankerte Kompetenzniveaus überführt werden, die zu erreichende Mindeststandards darstellen (vgl. Senkbeil, Schöber \& Ihme, 2019). Anhand der Kompetenzniveaus können die Studierendenanteile bestimmt werden, die zu verschiedenen Studienzeitpunkten über eine ausreichende ICT Literacy im Sinne von Mindeststandards verfügen.

\section{Theoretischer Hintergrund}

Um konkrete Untersuchungsannahmen formulieren zu können, wird zunächst kurz referiert, welche Rolle (digitale) Medienkompetenz im Rahmen von medienpädagogischen Kompetenzmodellen einnimmt. Darauf aufbauend wird dargestellt, wie sich die in den NEPS-Studien untersuchte ICT Literacy in erziehungswissenschaftliche Konzeptionen der Medienkompetenz einordnen lässt. Abschließend werden bisherige Befunde zur vermuteten Negativselektion von Lehramtsstudierenden in Bezug auf digitale Medien sowie zur Medienkompetenzförderung in der (universitären) Lehramtsausbildung zusammengefasst. 


\section{Strukturmodell der medienpädagogischen Kompetenz und der Medienkompetenz}

Für ein sachgerechtes und selbstbestimmtes Handeln und die Möglichkeit gesellschaftlicher Teilhabe in einer digitalisierten Welt müssen Kinder und Jugendliche erzieherisch unterstützt werden. Die allgemeine und bildungspolitische Diskussion um die schulische Förderung von digitaler Medienkompetenz hat sich durch das mittelmäßige Abschneiden deutscher Schülerinnen und Schüler in der internationalen Schulleistungsstudie ICILS 2013 (International Computer and Information Literacy Study) intensiviert (Eickelmann, 2017; Schiefner-Rohs \& Hofhues, 2018). In der Folge hat die Bildungspolitik Strategien initiiert, um den Erwerb digitaler Bildung flächendeckend in Bildung, Ausbildung und Fortbildung zu verankern (Bundesministerium für Bildung und Forschung [BMBF], 2016; Kultusministerkonferenz [KMK], 2016). Daraus ergeben sich u. a. besondere Anforderungen an die Ausbildung von Lehramtsstudierenden, die über entsprechende medienpädagogische Kompetenzen verfügen müssen, um digitale Medien im späteren Berufsalltag zielgerichtet und kompetenzorientiert in unterrichtliche Lehr-Lernprozesse einbeziehen zu können.

Die medienbezogenen Kompetenzanforderungen an Lehrpersonen lassen sich gemäß der Konzeptualisierung von Herzig et al. (2015) zu einem Strukturmodell mit folgenden drei Komponenten bündeln (siehe auch Abb. 1):

1. Mediendidaktische Kompetenz: Fähigkeit zur reflektierten Nutzung digitaler Medien zur Anregung und Unterstützung von Lernprozessen (z. B. Unterrichtsbeispiele mit digitalen Medien analysieren und einsetzen, z. B. zur Erkenntnisgewinnung);

2. Medienerzieherische Kompetenz: Förderung eines sachgerechten, selbstbestimmten und sozial verantwortlichen Handelns der Schülerinnen und Schüler mit digitalen Medien (z. B. eigenständige Auswahl und zielführende Nutzung von Medien)

3. Medienbezogene Schulentwicklungskompetenz: Fähigkeit, an der Gestaltung medienpädagogischer Konzepte für Schulen mitzuwirken.

Diesem Modell liegt ein Verständnis von Kompetenzen als erlernbare Leistungsdispositionen zugrunde. Diese Kompetenzen sind zur Bewältigung entsprechender Situationen im Lehrberuf erforderlich und sollten im Rahmen der universitären Ausbildungsphase erworben werden.

Eine notwendige Voraussetzung für den Erwerb medienpädagogischer Kompetenzen stellt die (eigene) Medienkompetenz dar (Herzig \& Martin, 2018; Tulodziecki, 2012). Diese beinhaltet die Fähigkeit und Bereitschaft, Medien und Informationstechnologien sachgerecht, selbstbestimmt, kreativ und sozial verantwortlich zu verwenden und lässt sich in folgende Bereiche untergliedern (Tulodziecki, 2012): Die ersten beiden Bereiche (B) umfassen zwei grundlegende Handlungsfelder von (digitalen) Medien in verschiedenen Kontexten (z. B. Information und Lernen, Austausch und Kooperation): (B1) Auswählen und Nutzen von Medienangeboten sowie (B2) die Gestaltung eigener Medienbeiträge. Um in den Handlungsfeldern kompetent agieren zu können, sind darüber hinaus Kenntnisse in folgenden Inhaltsbereichen erforderlich: (B3) das Verstehen und Bewerten von Mediengestaltungen (z. B. Darstellungsoder Interaktionsformen), (B4) das Erkennen und Bewerten von Medieneinfliussen (z. B. auf Realitätsvorstellungen und Wertorientierungen) und (B5) das Durchschauen und Bewerten von Bedingungen der Medienproduktion und-verbreitung (z. B. soziale, rechtliche Bedingungen). Eine Basis für alle Kompetenzbereiche stellen elementare Nutzungsformen digitaler Medien dar, womit technologische Basiskenntnisse und -fertigkeiten (TKF) gemeint sind, z. B. in Bezug auf gebräuchliche Office-Programme (Herzig \& Martin, 2017, 2018; Tulodziecki, 2015). 


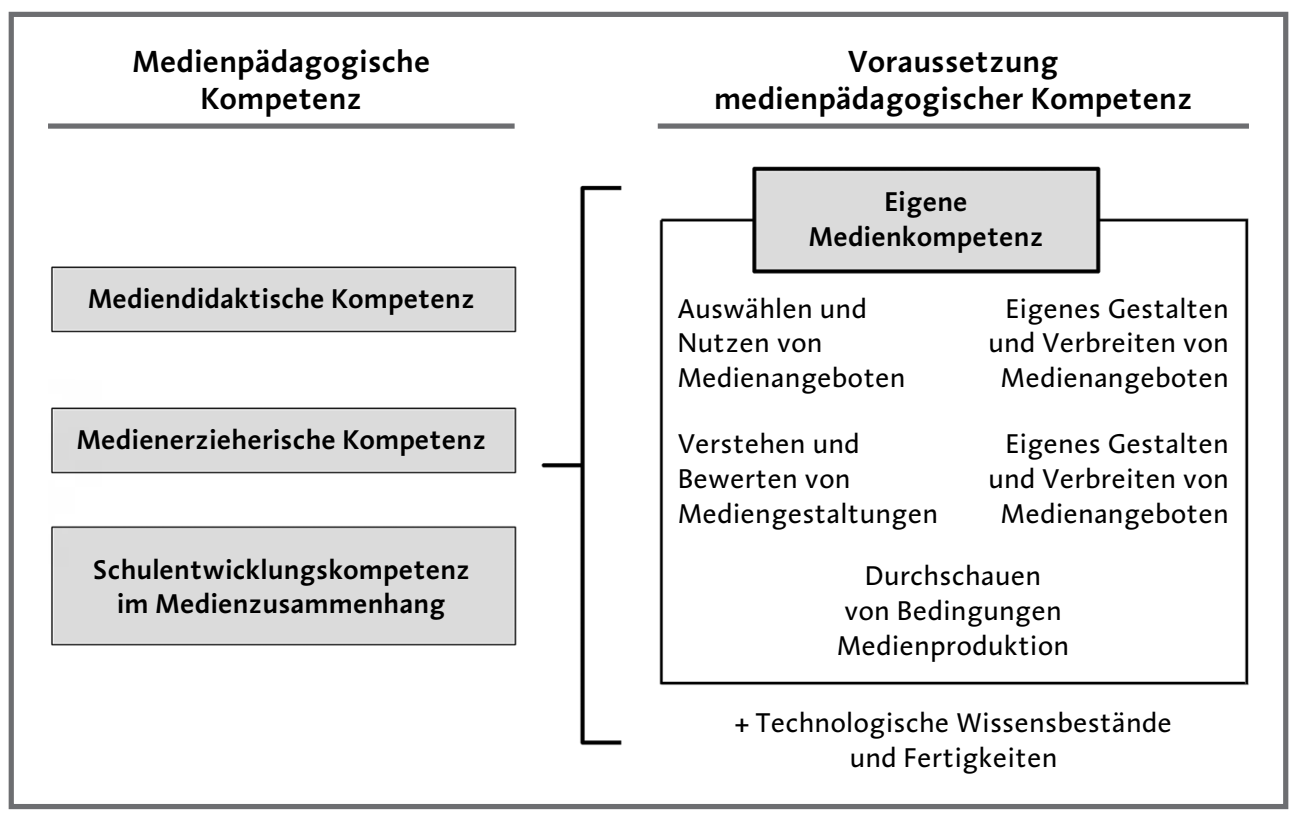

Abb. 1: Strukturmodell der medienpädagogischen Kompetenz in Anlehnung an Herzig et al. (2015).

\section{ICT Literacy als Teilkonstrukt der Medienkompetenz}

Befunde zur Medienkompetenz von (angehenden) Lehrkräften anhand objektiver Testdaten liegen kaum vor (Hermida, Hielscher \& Petko, 2017; Herzig \& Martin, 2017). Andererseits weisen die von der Medienpädagogik entwickelten Konzepte eine hohe Affinität zu Konzeptualisierungen der ICT Literacy im Bereich der empirischen Bildungsforschung auf (z. B. Fraillon, Schulz \& Ainley, 2013; Senkbeil, Ihme \& Wittwer, 2013). Entsprechend decken die in diesen Studien (z. B. ICILS, 2013; NEPS) erfassten Kompetenzen zumindest Teilbereiche einer umfassenden Medienkompetenz ab (vgl. Eickelmann, 2017; Hermida et al., 2017; Herzig \& Martin, 2017), wie nachfolgend am Beispiel der ICT Literacy-Tests in NEPS für Studierende gezeigt wird.

In NEPS wird die ICT Literacy von Studierenden gemäß eines Multikohorten-SequenzDesigns (Blossfeld, Roßbach \& von Maurice, 2011) zu verschiedenen Zeitpunkten und in verschiedenen Startkohorten (Starting Cohort,
SC) erhoben. Analog zur medienpädagogischen Kompetenz und Medienkompetenz wird in NEPS unter ICT Literacy eine erlernbare kognitive Leistungsdisposition verstanden (Klieme, Hartig \& Rauch, 2008). Diese umfasst funktionale (d. h. von der Lebens- und Arbeitswelt ausgehende) Wissensbestände und Fertigkeiten im Umgang mit digitalen Medien, die für eine erfolgreiche Teilhabe an der Gesellschaft und für ein kontinuierliches Weiterlernen bedeutsam sind. Hierzu zählen neben technologischer Kompetenz (grundlegendem Funktionswissen über Hardware und Programmanwendungen) vor allem Aspekte der Informationskompetenz. Diese beinhaltet die Fähigkeit, mithilfe digitaler Medien Informationen zu ermitteln, diese kritisch auszuwählen und sie effektiv zu nutzen. ICT Literacy konstituiert sich aus dem Zusammenwirken von kognitiven und technologischen Kompetenzen und wird entsprechend als eindimensionales Konstrukt im Sinne einer domänenübergreifenden Schlüsselkompetenz aufgefasst (Senkbeil et al., 2013). 
Die NEPS ICT Literacy-Rahmenkonzeption differenziert fünf Prozesskomponenten (PK), welche die für eine zielgerichtete Nutzung digitaler Medien benötigten Wissensbestände und Fertigkeiten beschreiben:

- PK1, Anwenden und Verstehen: Grundlegende Kenntnisse über Programmanwendungen sowie Operationen, um auf Informationen zuzugreifen (z. B. Textdokument formatieren);

- PK2, Suchen und Organisieren: Fähigkeit, Informationen effizient zu ermitteln (z. B. adäquate Suchbegriffe eingeben) oder Informationen miteinander nach spezifischen Kriterien zu vergleichen (z. B. Datensätze sortieren);

- PK3, Bewerten: Informationen hinsichtlich verschiedener Kriterien wie z. B. Glaubwürdigkeit prüfen;
- PK4, Erzeugen: Fähigkeit, Dokumente und Dateien zu bearbeiten oder zu erstellen (z. B. Tabellen anlegen, Präsentationen erstellen);

- PK5, Kommunizieren: Informationen verständlich und adressatengerecht übermitteln (z. B. informative und verständliche E-Mails schreiben oder Grafiken erstellen).

Die Messung von ICT Literacy in NEPS berücksichtigt gebräuchliche Programmanwendungen (Textverarbeitung, Tabellenkalkulation, Präsentation, E-Mail, internetgestützte Suchmaschinen und Datenbanken) und erfolgt anhand persönlicher (z. B. Informationssuche) und bildungsbezogener Kontexte (z. B. Lernen). Insgesamt lassen sich folgende Überlappungen zwischen den Handlungsfeldern der Medienkompetenz und der Rahmenkonzeption in NEPS feststellen (siehe auch Abb. 2):

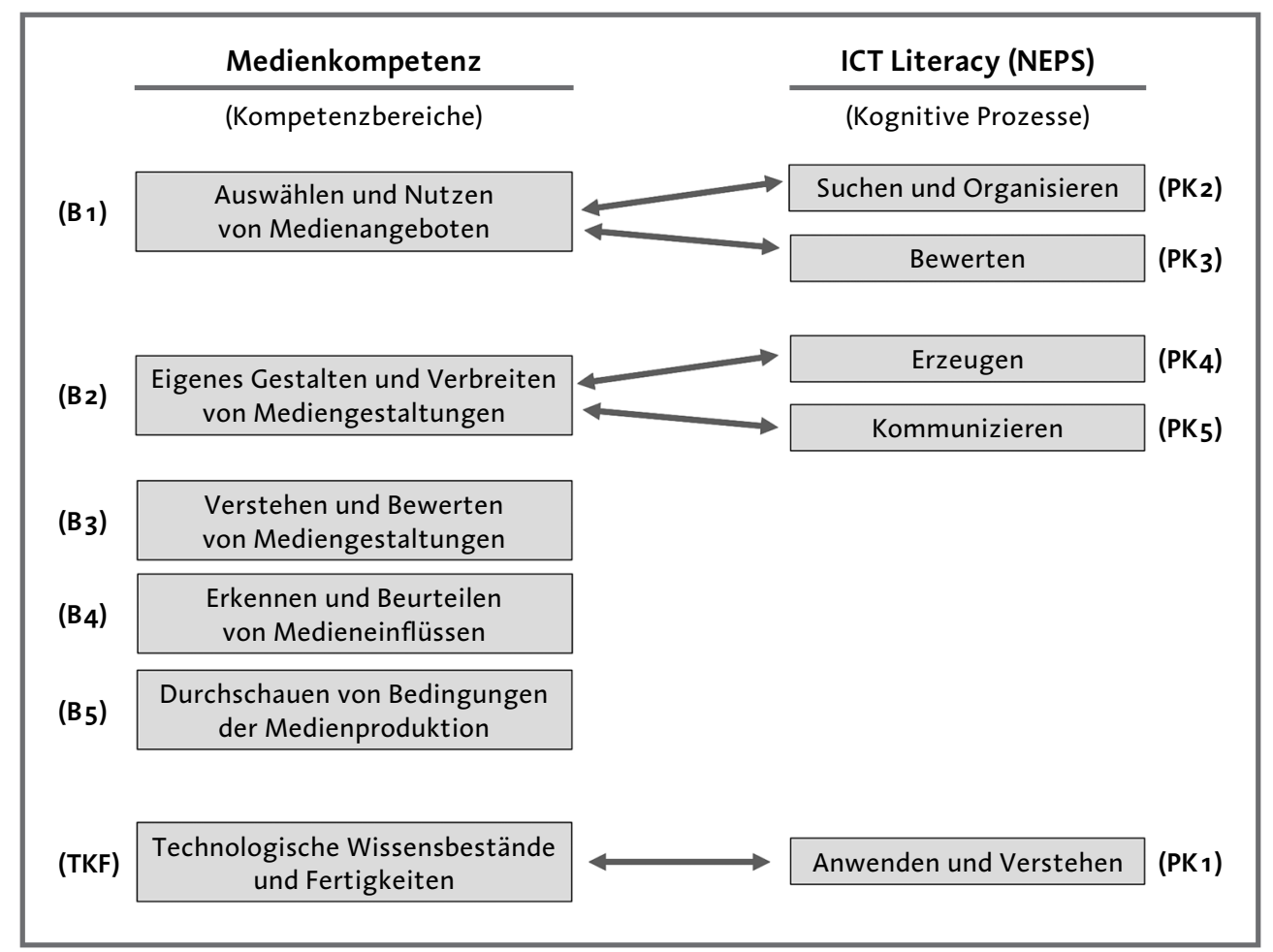

Abb. 2: Vergleich von Medienkompetenz (in Anlehnung an Herzig \& Martin, 2017) und ICT LiteracyRahmenkonzeption in NEPS (Senkbeil et al., 2013). 
1. Die Auswahl und Nutzung verschiedener Medienangebote (B1) beinhaltet unter anderem die Fähigkeiten zur effizienten Informationsbeschaffung mit Suchmaschinen (entspricht PK2 in NEPS) und zur kriterienbezogenen Einschätzung der Nützlichkeit von Medienangeboten, z. B. im Rahmen einer Internetrecherche (entspricht PK3). Weitere Beispiele beschreiben die Fähigkeit, Tabellenkalkulationsprogramme zum Zweck der Problemlösung zu verwenden (entspricht PK2) oder bei der Kommunikation mit anderen Personen unter verschiedenen Möglichkeiten (z. B. E-Mail, Chat, persönliches Gespräch) sinnvoll auszuwählen (entspricht PK3).

2. Bei der Gestaltung und Verbreitung eigener Medienbeiträge (B2) werden als Kompetenzaspekte beispielsweise das Erstellen von Medienprodukten wie Texte oder Präsentationen (entspricht PK4), das adressatengerechte Kommunizieren (PK5) oder das Erstellen einer multimedialen Webseite unter Nutzung von z. B. Texten und Bildern (entspricht PK5) genannt (vgl. hierzu Herzig, 2014; Tulodziecki, 2010, 2015).

3. Technologische Basiskenntnisse und -fertigkeiten wie der kompetente Umgang mit Office-Programmen (z. B. Formatieren eines Textdokuments) oder das Verstehen und Anlegen von Ordnerstrukturen entsprechen PK1 in NEPS (Herzig \& Martin, 2017; Tulodziecki, 2015).

Die Gemeinsamkeiten zusammenfassend deckt ICT Literacy gemäß der Rahmenkonzeption in NEPS einen Teilbereich des umfassenderen Konstrukts der Medienkompetenz ab und kann somit als Teilkonstrukt der Medienkompetenz aufgefasst werden. Vor diesem Hintergrund eignen sich die in NEPS eingesetzten ICT Literacy-Tests, wichtige Teilbereiche dieses Konstrukts, das eine notwendige Voraussetzung für den Erwerb medienpädagogischer Kompetenz darstellt, bei angehenden Lehrkräften zu überprüfen.

\section{Negative Selektion von Lehramtsstudierenden in Bezug auf digitale Medien}

In der Forschungsliteratur finden sich vereinzelt Hinweise auf eine Negativselektion von Lehramtsstudierenden in Bezug auf ihre digitale Medienkompetenz. Danach rekrutieren sich Lehramtsstudierende aus Abiturientinnen und Abiturienten mit vergleichsweise distanzierten Haltungen zu digitalen Medien und Kompetenzdefiziten gegenüber Studierenden anderer Fachrichtungen. Als Erklärungsansatz dient der „Teufelskreis fehlender Medienbildung“ (Kammerl \& Pannarale, 2007), wonach die ICT-bezogenen Defizite angehender Lehramtsstudierender Folge ihres medialen Habitus sind. Dieser zeichnet sich durch eine vergleichsweise große Distanz zu digitalen Medien aus, die auf familiär geprägte Mediennutzungsmuster und die elterliche Medienerziehung, z. B. eine äußerst kritische Haltung der Eltern gegenüber digitalen Medien und eine starke Reglementierung der Nutzung digitaler Medien, zurückzuführen ist (Kommer \& Biermann, 2012). Da auch im Studium keine ausreichende Ausbildung im Bereich der Medienkompetenzförderung erfolgt und sie als angehende Lehrkräfte in der Regel auf eine Schulpraxis stoßen, in der die digitale Medienbildung im Unterricht eher vernachlässigt wird, beginnt der Kreislauf somit von Neuem.

Die empirische Befundlage zur These distanzierter Haltungen gegenüber digitalen Medien und damit einhergehenden Kompetenzdefiziten von Lehramtsstudierenden ist jedoch vergleichsweise dünn und beruht auf Selbsteinschätzungen, aber keinen objektiv erhobenen Kompetenzmaßen (Kammerl \& Mayrberger, 2011). Hingegen wird die Annahme einer unzureichenden Medienkompetenzförderung in der ersten Phase der Lehramtsausbildung durch aktuelle Befunde gestützt. Trotz vieler Bestrebungen ist eine flächendeckende Verankerung der Medienpädagogik in der universitären Phase der Lehrerbildung in Deutschland bis heute nicht gelungen (Eickelmann, Lorenz \& Endberg, 2016). Medienbezogene Themen sind nicht gleichermaßen in allen Lehrplänen der Bundesländer berück- 
sichtigt (Kammerl \& Mayrberger, 2011), und es besteht immer noch ein sehr geringer Verpflichtungsgrad für Lehramtsstudierende, sich während des Studiums mit digitalen Medien auseinanderzusetzen. Beispielsweise sieht nur die Hälfte der Hochschulen verpflichtende Lehrangebote zum Erwerb digitaler Medienkompetenz in den Lehramtsstudiengängen vor, die zudem nur für einzelne Lehramtsfächer gelten (Bertelsmann Stiftung, CHE Centrum für Hochschulentwicklung, Deutsche Telekom Stiftung \& Stifterverband für die Deutsche Wissenschaft 2018). Entsprechend wird von mehr als $80 \%$ der Lehrkräfte der Sekundarstufe I in Deutschland eine stärkere Vorbereitung auf den schulischen Medieneinsatz in der universitären Lehrerausbildung befürwortet und für notwendig gehalten (Eickelmann et al., 2016). Vor diesem Hintergrund ist zu befürchten, dass potenzielle Defizite der Lehramtsstudierenden in der ICT Literacy zu Studienbeginn auch im Verlauf des Studiums nicht mehr kompensiert werden können (Herzig \& Grafe, 2007).

Darüber hinaus sind im Sinne einer negativen Binnenselektion Defizite in der ICT Literacy für diejenigen Lehramtsstudierenden zu vermuten, die kein MINT (Mathematik, Informatik, Naturwissenschaften und Technik)-Fach gewählt haben (vgl. hierzu Eickelmann, Lorenz \& Endberg, 2017). Für angehende Lehrkräfte mit gewähltem MINT-Fach wird eine vergleichsweise hohe Technikaffinität angenommen, die sich in höheren positiveren Einstellungen gegenüber digitalen Medien und einer höheren ICT Literacy als bei Lehramtsstudierenden ohne MINT-Fach widerspiegeln sollte (z. B. Barak, 2014). Diese Annahme beruht auf der Überlegung, dass die MINT-Disziplinen die Grundlage der Digitalisierung bilden und somit für das Verständnis einer zunehmend digitalisierten Welt notwendig sind. Weiterhin weisen die MINTFächer durch techniknahe Themen und eine Vielzahl von Anwendungsmöglichkeiten digitaler Medien ein besonders hohes Potenzial für deren wirkungsvollen Einsatz im Unterricht auf (Eickelmann et al., 2017). Auch dieser Annahme mangelt es an stützenden empirischen Befunden.

\section{Fragestellungen}

Anhand einer Sekundäranalyse von Daten, die in NEPS an zwei Startkohorten (SC4, SC5) erhoben wurden, wird die ICT Literacy von angehenden und fortgeschrittenen Lehramtsstudierenden (sechstes Fachsemester) untersucht. Basierend auf den referierten Überlegungen ergeben sich folgende Fragestellungen und $\mathrm{Hy}$ pothesen $(\mathrm{H})$.

\section{Fragestellung 1: Negative Selektion von Lehramtsstudierenden}

In Anlehnung an die These zum „Teufelskreis fehlender Medienbildung " (Kammerl \& Mayrberger, 2011) wird geprüft, ob Lehramtsstudierende über eine geringere ICT Literacy verfügen als Studierende anderer Fachrichtungen ( $\mathrm{H} 1 \mathrm{a}$ : angehende Studierende, H1 b: fortgeschrittene Studierende). Gemäß der Überlegung zu negativen Binnenselektionseffekten (z. B. Barak, 2014) wird darüber hinaus die Hypothese geprüft, ob Lehramtsstudierende, die kein MINTFach gewählt haben, über eine geringere ICT Literacy als Lehramtsstudierende mit mindestens einem MINT-Fach verfügen (H1 c: angehende Studierende, H1 d: fortgeschrittene Studierende).

\section{Fragestellung 2: Entwicklung der ICT Literacy von Lehramts- und anderen Studierenden}

Mit dieser Fragestellung wird geprüft, inwieweit Lehramtsstudierende im Verlauf des ersten Studienabschnitts die vermuteten Defizite in der ICT Literacy im Vergleich zu Studierenden anderer Fachrichtungen ausgleichen können. Aufgrund der unzureichenden Medienkompetenzförderung in der ersten Ausbildungsphase (z. B. Eickelmann et al., 2016) kann vermutet werden, dass sich Kompetenzdefizite über das Studium sogar noch vergrößern (Hypothese $2 \mathrm{a}, \mathrm{H} 2 \mathrm{a}$ ). Analog zu den Hypothesen $\mathrm{H} 1 \mathrm{c}$ und $\mathrm{H} 1 \mathrm{~d}$ wird zusätzlich geprüft, ob ebenfalls die vermuteten Kompetenzdefizite von Lehramtsstudierenden 
ohne MINT-Fach im Vergleich zu Lehramtsstudierenden mit MINT-Fach über die Zeit noch zunehmen (Hypothese $2 \mathrm{~b}, \mathrm{H} 2 \mathrm{~b}$ ). Da der vorliegenden Arbeit nur zwei querschnittliche, aber keine längsschnittlichen Datensätze (SC4, SC5) zugrunde liegen, die zudem unterschiedlichen Kohorten angehören (Geburtsjahrgänge SC4: 1993 bis 1998, Median = 1996; Geburtsjahrgänge SC5: 1988 bis 1993; Median = 1990), kann die Entwicklung der ICT Literacy für die genannten Gruppen nur geschätzt werden. Um eine möglichst unverzerrte Schätzung zu erhalten, werden mögliche Unterschiede in den Eingangsvoraussetzungen zwischen den Startkohorten kontrolliert. Als Kontrollvariablen fungieren Geschlecht, allgemeine kognitive Fähigkeit und elterliches Bildungsniveau.

\section{Fragestellung 3: Kompetenzniveaus von angehenden und fortgeschrittenen Lehramtsstudierenden}

Abschließend werden auf Basis kriterial verankerter Kompetenzniveaus die Anteile von Lehramtsstudierenden bestimmt, die zu verschiedenen Studienzeitpunkten (Studienbeginn, sechstes Fachsemester) über eine ausreichende ICT Literacy im Sinne von Mindeststandards verfügen (siehe auch Abschnitt „Standard-Setting-Verfahren" im Methodenteil). Angesichts empirischer Hinweise, die auf eine vergleichsweise schwache ICT Literacy von angehenden und aktuellen Lehrkräften hindeuten (z. B. Lorenz et al., 2016; Zylka \& Müller, 2011), und in Anlehnung an den „Teufelskreis fehlender Medienbildung" sind substanzielle Anteile (>20\%) angehender und fortgeschrittener Lehramtsstudierender (Hypothesen 3a und $3 \mathrm{~b}, \mathrm{H} 3 \mathrm{a}$ und $\mathrm{H} 3 \mathrm{~b}$ ) zu erwarten, die den jeweiligen Mindeststandard nicht erreichen. Gemäß den Hypothesen 1 und 2 sollten diese Anteile bei Lehramtsstudierenden größer ausfallen als bei Studierenden anderer Fachrichtungen (Hypothese $3 \mathrm{c}, \mathrm{H} 3 \mathrm{c}$ : angehende Studierende; Hypothese $3 \mathrm{~d}, \mathrm{H} 3 \mathrm{~d}$ : fortgeschrittene Studierende).

\section{Methode}

Die empirische Datengrundlage für die vorliegende Arbeit bildeten Erhebungen in NEPS. Die Anlage und Durchführung von NEPS ist detailliert in Blossfeld et al. (2011) beschrieben. Wir beschränken uns daher im Folgenden auf die für die vorliegende Arbeit wesentlichen Informationen. Diese umfassen die Beschreibung der Stichproben, der ICT-LiteracyTests und der Linking-Studie, mit der die ICT-Literacy-Tests beider Studierendengruppen auf einer gemeinsamen Metrik skaliert wurden, sowie des Standard-Setting-Verfahrens zur Generierung kategorialer Kompetenzniveaus.

\section{Stichproben}

Die Fragestellungen wurden mithilfe der Daten aus der SC4 (angehende Studierende) und der SC5 (fortgeschrittene Studierende) des Nationalen Bildungspanels bearbeitet. Die ICT-Literacy-Tests wurden in beiden Kohorten im Jahr 2013 durchgeführt. Die Personen der SC4 befanden sich zu dem Zeitpunkt in der 12. Klassenstufe, die der SC5 im sechsten Fachsemester. Für die Analysen berücksichtigt wurden diejenigen Studierenden, für die ICT-LiteracyTestwerte sowie gültige Angaben zur Aufnahme eines Studiums und zum gewählten Studienfach (z. B. Lehramt vs. Nicht-Lehramt) vorliegen. Die Stichprobe der SC4 umfasst alle Personen, die im Jahr der Erlangung der Hochschulzugangsberechtigung ein Studium aufgenommen haben $(N=1518 ; 50,7 \%$ weiblich; Alter: $M=17.56, S D=0.58)$. Um eine zur SC4 vergleichbare Stichprobe zu generieren, beinhaltet die Stichprobe der SC5 ebenfalls die Studierenden, die direkt nach Erreichen ihrer Hochschulzugangsberechtigung ein Studium begonnen haben $(N=1766,49,5 \%$ weiblich; Alter: $M=23.06, S D=$ 0.97). Um repräsentative Ergebnisse zu erhalten, wurden die Daten entsprechend den Populationsverteilungen gewichtet. Tabelle 1 zeigt die Verteilung der Studierenden beider Kohorten nach Studiengang (Lehramt vs. Nicht-Lehramt) und Fächerkombination (MINT-Fach vs. kein MINT-Fach) in absoluten (ungewichtet) und prozentualen Häufigkeiten (gewichtet). Erwartungskonform wird das Lehramtsstudium häufiger von Frauen gewählt (SC4: 68,0\%; SC5: 66,7\%). Die deutlich größere Fallzahl an Lehramtsstudierenden in der SC5 resultiert aus einem Oversampling dieser Gruppe in dieser Startkohorte. 
Tab. 1: Verteilung der angehenden und fortgeschrittenen Studierenden nach Studiengruppe und Fächerkombination

\begin{tabular}{|c|c|c|c|c|c|c|c|}
\hline \multirow[b]{2}{*}{ Studiengang } & \multirow[b]{2}{*}{ Fachgruppe } & \multicolumn{3}{|c|}{$\begin{array}{c}\text { Angehende } \\
\left.\text { Studierende ( } \mathrm{SC}_{4}\right)\end{array}$} & \multicolumn{3}{|c|}{$\begin{array}{l}\text { Fortgeschrittene } \\
\text { Studierende }\left(\mathrm{SC}_{5}\right)\end{array}$} \\
\hline & & $n$ & \% (gew.) & $\%(w)$ & $n$ & \% (gew.) & $\%(w)$ \\
\hline $\begin{array}{l}\text { Lehramt } \\
\text { Nicht Lehramt }\end{array}$ & & $\begin{array}{r}221 \\
1297\end{array}$ & $\begin{array}{l}13.3 \\
86.7\end{array}$ & $\begin{array}{l}68.0 \\
47.6\end{array}$ & $\begin{array}{r}748 \\
1018\end{array}$ & $\begin{array}{l}14.6 \\
85.4\end{array}$ & $\begin{array}{l}66.7 \\
43.5\end{array}$ \\
\hline Lehramt (100\%) & $\begin{array}{l}\text { MINT-Fach } \\
\text { Kein MINT-Fach }\end{array}$ & $\begin{array}{r}71 \\
150\end{array}$ & $\begin{array}{l}45 \cdot 5 \\
55 \cdot 5\end{array}$ & $\begin{array}{l}65.1 \\
69.3\end{array}$ & $\begin{array}{l}242 \\
506\end{array}$ & $\begin{array}{l}33.6 \\
66.4\end{array}$ & $\begin{array}{l}55 \cdot 3 \\
72.3\end{array}$ \\
\hline Nicht Lehramt (100\%) & $\begin{array}{l}\text { MINT-Fach } \\
\text { Kein MINT-Fach }\end{array}$ & $\begin{array}{l}615 \\
682\end{array}$ & $\begin{array}{l}46.6 \\
53.4\end{array}$ & $\begin{array}{l}33.2 \\
60.2\end{array}$ & $\begin{array}{l}460 \\
558\end{array}$ & $\begin{array}{l}47.5 \\
52.5\end{array}$ & $\begin{array}{l}27.2 \\
58.3\end{array}$ \\
\hline
\end{tabular}

Anmerkung: $n=$ Fallzahl in Stichprobe; \% (gew.)=prozentualer Anteil gemäß Populationsverteilung auf Grundlage der Fächersystematik des Statistischen Bundesamts; \% (w)=prozentualer Anteil weiblicher Studierender.

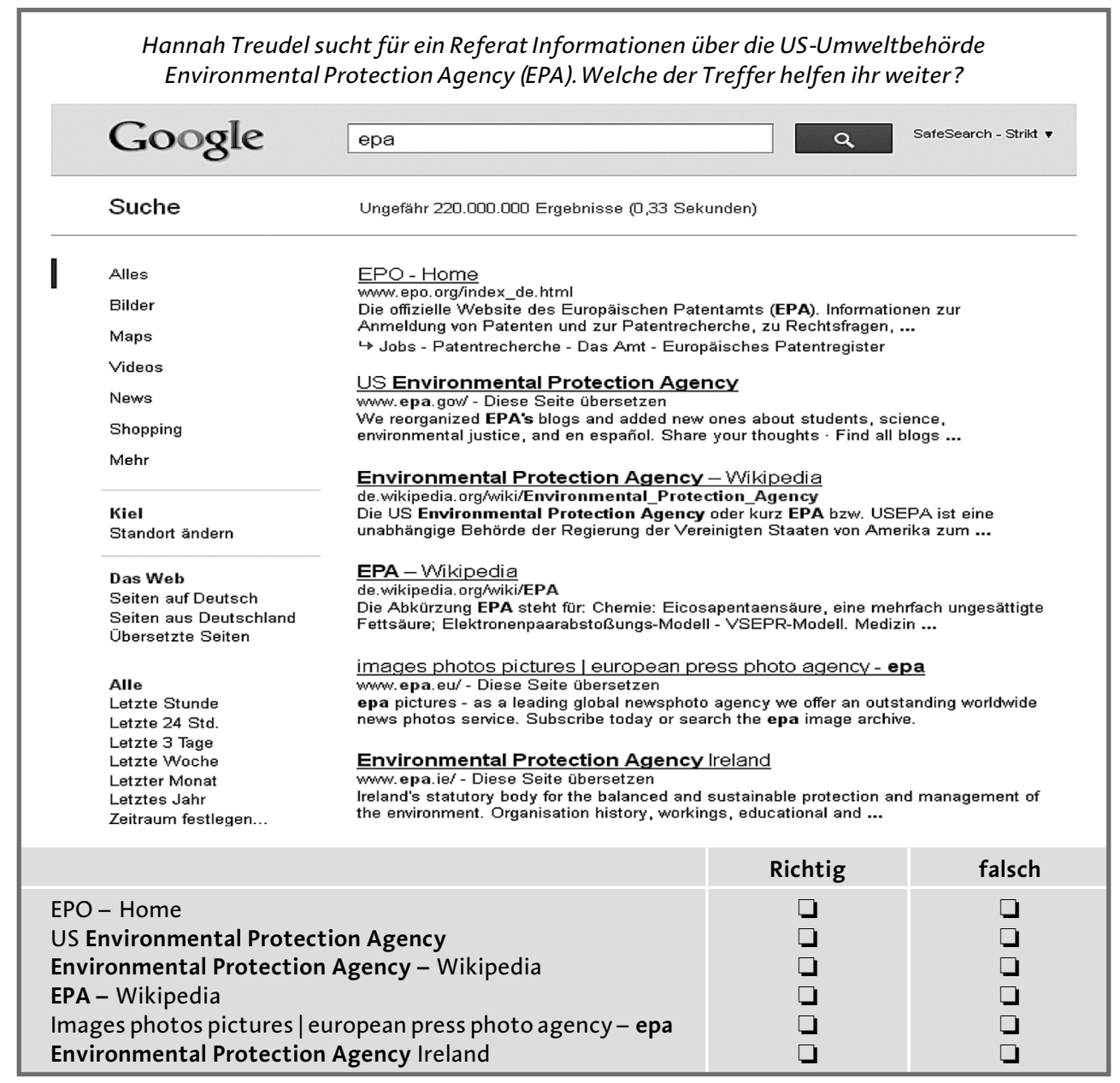

Abb. 3: Beispielitem zur Prozesskomponente Bewertung (Programmanwendung: Internet); Kompetenzniveau Basic (476 Punkte). 
10 Martin Senkbeil, Jan Marten Ihme, Christian Schöber

\section{Instrumente}

\section{ICT Literacy-Tests}

Beide Tests, die bei den Studierenden eingesetzt wurden, umfassen bei einer Bearbeitungszeit von 30 Minuten jeweils 30 MC-Aufgaben. Die Aufgaben enthalten authentische Problemstellungen und erfassen, ob die Testpersonen angemessen mit bestimmten Aufgabenstellungen umgehen können. Für eine möglichst realitätsnahe Gestaltung werden in den Aufgabenstimulus Screenshots integriert (z. B. von einem Internet-Browser) und als Distraktoren häufig realistische Antwortalternativen in Form von Schaltflächen oder Menüs vorgegeben (siehe Abb. 3 und 4 für Beispielitems).
Durch eine in NEPS implementierte LinkingStudie konnten die beiden Kompetenztests gemeinsam skaliert werden (vgl. Pohl \& Carstensen, 2013). In dieser bearbeiteten insgesamt $N=394$ Schülerinnen und Schüler der 12. Klasse aus vier Bundesländern sowohl den Test für Klasse 12 (SC4) als auch den Test für die fortgeschrittenen Studierenden (SC5). Die insgesamt 60 Items beider Tests wurden unter Berücksichtigung der drei Datenquellen (LinkingStichprobe, SC4, SC5) mittels eines eindimensionalen Raschmodells in ConQuest (Wu, Adams, Wilson $\&$ Haldane, 2007) unter Verwendung eines Hintergrundmodells (Fächergruppe, Geschlecht, kognitive Grundfähigkeiten, sozialer Hintergrund) gemeinsam

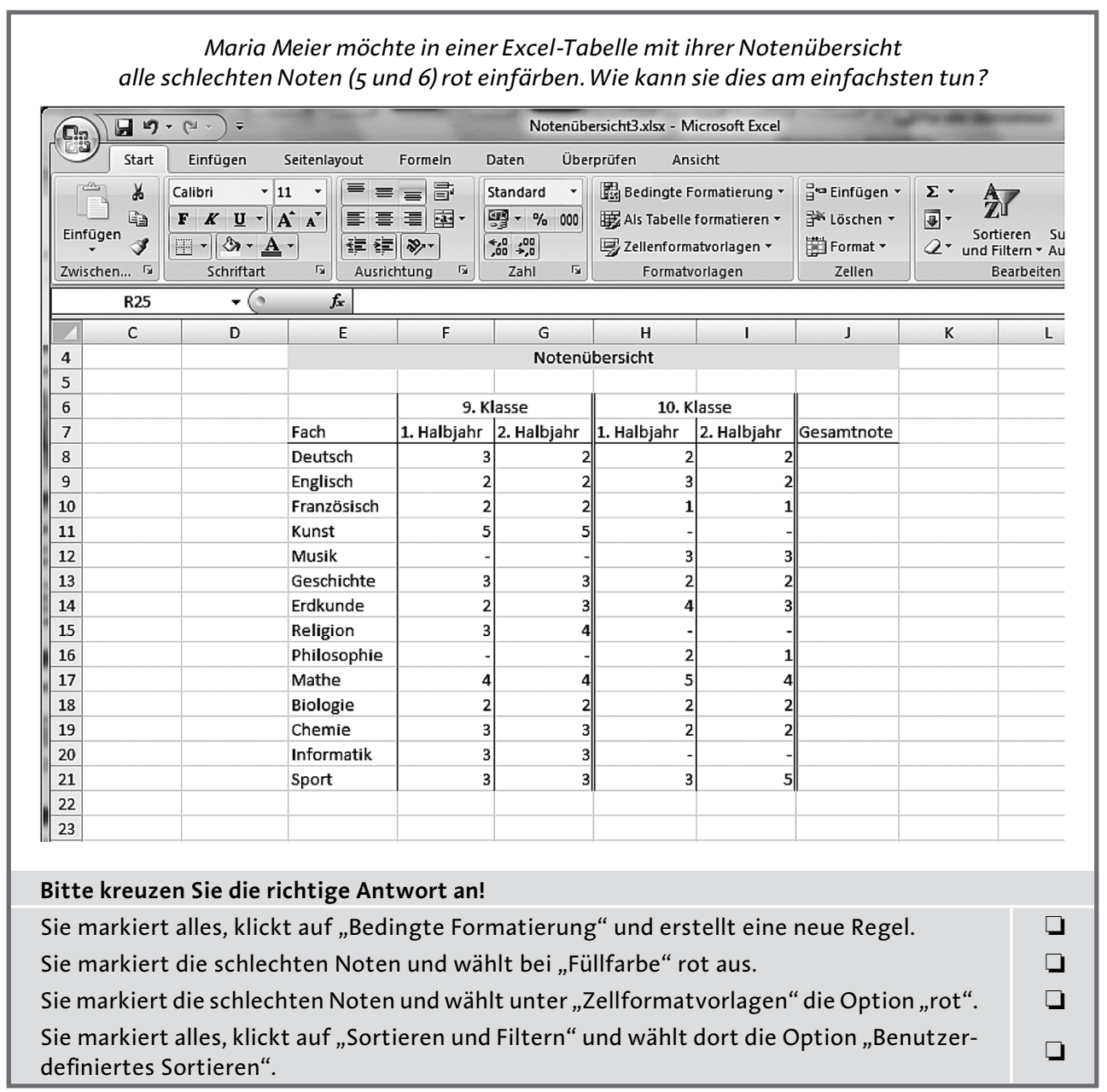

Abb. 4: Beispielitem zur Prozesskomponente Suchen und Organisieren (Programmanwendung: Tabellenkalkulation); Kompetenzniveau Proficient (663 Punkte). 
skaliert. Die auf diese Weise ermittelten Kompetenzwerte (fünf Plausible Values) wurden in der gewichteten Stichprobe der fortgeschrittenen Studierenden auf einen Mittelwert von $M=500 \mathrm{mit} S D=100$ transformiert. Die EAP/PV-Reliabilität des Gesamttests beträgt .72 und ist somit als ausreichend anzusehen (Rost, 2004).

\section{Kognitive Grundfähigkeiten}

Zur Erfassung der kognitiven Grundfähigkeiten wird in NEPS ein sprachfreier Matrizentest zum schlussfolgernden Denken (NEPS-MAT; Haberkorn \& Pohl, 2013) eingesetzt. Dieser umfasst 12 Aufgaben bei einer Bearbeitungszeit von 9 Minuten und besitzt eine ausreichende Reliabilität (Cronbachs $\alpha=.73$ ). Der individuelle Leistungswert ergibt sich durch den Summenscore über alle 12 Items.

\section{Sozialer Hintergrund}

Als Indikator für den sozialen Hintergrund der Studierenden wurde der höchste elterliche Schul- und Ausbildungsabschluss zugrunde gelegt, da dieser die Auswahl eines bestimmten Studienfachs stark beeinflusst (Reimer \& Pollak, 2009). Anhand der in NEPS verfügbaren Comparative Analysis of Social Mobility in Industrial Nations-Klassifikation (CASMIN; Brauns, Scherer \& Steinmann, 2003) wurden diese Angaben in die Anzahl der Bildungsjahre umgerechnet.

\section{Standard-Setting-Verfahren}

Um Kompetenzniveaus für beide Studierendengruppen zu generieren, wurde ein Standard-Setting-Verfahren (Pant, Tiffin-Richards \& Köller, 2010) auf Grundlage aller gemeinsam skalierten Items durchgeführt. In diesem bestimmte ein Expertenpanel aus den Bereichen ICT Literacy und digitale Hochschullehre diejenigen Wissensbestände und Fertigkeiten, über die eine Person für ein bestimmtes Kompetenzniveau verfügen muss (siehe Senkbeil et al., 2019, für eine ausführliche Darstellung). Als Ergebnis dieses Vorgehens wurden zwei Kompetenzniveaus im Sinne von Mindeststandards definiert. Das Kompetenzniveau Basic definiert ein digitales Grundbildungsniveau, das für die Aufnahme eines Studiums notwendig ist und den Erwerb weiterer, studien- und berufsrelevanter Kompetenzen ermöglicht. Das Kompetenzniveau Proficient beschreibt ein erweitertes Grundbildungsniveau, welches Studierende im Ver- lauf ihres Studiums im Hinblick auf spätere berufliche Anforderungen erwerben sollten. Darüber hinaus wurde das Kompetenzniveau Below Basic festgelegt. Studierende auf diesem Niveau erfüllen nicht die für die Aufnahme eines Studiums erforderlichen Mindestanforderungen und verfügen nur über rudimentäre Wissensbestände und Fertigkeiten (z. B. grundlegende Probleme bei der Identifizierung, Bewertung und Weiterverarbeitung ermittelter Informationen).

\section{Analysen}

Für die Analysen zur Beantwortung der Hypothesen wurde die Statistiksoftware SPSS 24 verwendet. Für Fragestellung 1 wurden zunächst die mittleren Kompetenzwerte unter Berücksichtigung der Populationsgewichte für die jeweilige Studierendengruppe berechnet. Da die Mittelwerte und andere statistische Kennwerte auf fünf PVs beruhen, wurden alle Analysen fünfmal durchgeführt und die Ergebnisse gemäß den Vorschlägen von Rubin (Rubin's Rules; Rubin, 1987) gemittelt. Die Zuordnung der Studienfächer zu den Fachgruppen (MINT-Fach vs. kein MINT-Fach) erfolgte auf Grundlage der Kategorisierung von Beutenagel, Dittmann, Meyer und Baumhauer (2018). Die inferenzstatistische Prüfung erfolgte für jede Startkohorte anhand einer zweifaktoriellen Varianzanalyse mit den Faktoren Studiengang (Lehramt vs. kein Lehramt) und Fachgruppe sowie anschließenden Post-Hoc-Tests für Einzelvergleiche (Fragestellung 1).

Für die geschätzte Entwicklung der ICT Literacy und die inferenzstatistische Prüfung von Gruppenunterschieden wurde eine dreifaktorielle Varianzanalyse mit dem zusätzlichen Faktor Startkohorte durchgeführt. Als Kontrollvariablen wurden zusätzlich Geschlecht, allgemeine kognitive Fähigkeit und elterliches Bildungsniveau aufgenommen, um mögliche Unterschiede in den Eingangsvoraussetzungen zwischen den Startkohorten zu kontrollieren und angesichts des Fehlens eines echten Längsschnittdesigns - eine möglichst unverzerrte Schätzung der Kompetenzentwicklung über die erste Ausbildungsphase zu ermöglichen (Fragestellung 2). Abschließend wurden mit den Schwellenwerten aus dem Standard-Setting-Verfahren die Verteilungen auf den Kompetenzniveaus berechnet (Fragestellung 3). Für die Prüfung von Verteilungsunterschieden in Abhängigkeit vom Studiengang wurde der $\chi^{2}$-Test verwendet. 
12 Martin Senkbeil, Jan Marten Ihme, Christian Schöber

\section{Ergebnisse}

\section{ICT Literacy der Lehramtsstudierenden (Fragestellung 1)}

Die inferenzstatistischen Ergebnisse der Varianzanalysen sind Tabelle 2 zu entnehmen. Die deskriptiven Ergebnisse zur ICT Literacy sowie die Resultate der Einzelvergleiche sind in Tabelle 3 dargestellt. Die Varianzanalysen weisen hypothesenkonform für beide Studierendengruppen auf signifikante Unterschiede in der ICT Literacy zwischen den Studiengängen hin (siehe signifikante Haupteffekte für Studiengang in Tab. 2). Die Unterschiede entsprechen nahezu durchgehend kleinen Effektstärken. Dabei ergeben sich erwartungskonform signifikante Kompetenzrückstande (siehe V1, Tab. 3) sowohl für die angehenden Lehramtsstudierenden (30 Punkte, $d=0.32 ; \mathrm{H} 1 \mathrm{a}$ ) als auch für die fortgeschrittenen Lehramtsstudierenden (47 Punkte, $d=0.47$; H1 b).

Ebenso lassen sich gemäß der vermuteten negativen Binnenselektion bei beiden Startkohorten signifikante Haupteffekte in Abhängigkeit von der Fachgruppe (MINT- vs. kein MINT-Fach) ermitteln. Bei drei der vier Einzelvergleiche lassen sich die Mittelwertdifferenzen statistisch absichern (siehe V2 und V3, Tab. 3). Die Kompetenzrückstände der Studierenden ohne MINT-Fach betragen zwischen 26 und 39 Punkten und entsprechen einem kleinen Effekt $(0.20 \leq d \leq 0.49)$. Nur der Unterschied bei den angehenden Lehramtsstudierenden (26 Punkte, $d=0.30$ ) wird aufgrund der

Tab. 2: Ergebnisse der zweifaktoriellen Varianzanalysen für die angehenden und fortgeschrittenen Studierenden

\begin{tabular}{|c|c|c|c|c|c|c|}
\hline \multirow[b]{2}{*}{ Quelle } & \multicolumn{3}{|c|}{$\begin{array}{c}\text { Angehende } \\
\left.\text { Studierende ( } \mathrm{SC}_{4}\right)\end{array}$} & \multicolumn{3}{|c|}{$\begin{array}{l}\text { Fortgeschrittene } \\
\text { Studierende }\left(\mathrm{SC}_{5}\right)\end{array}$} \\
\hline & $F$ & $d f$ & Eta $^{2}$ & $F$ & $d f$ & $\mathrm{Eta}^{2}$ \\
\hline Korrigiertes Modell & $19.89^{\star *}$ & 3,1517 & .04 & $55.46^{* *}$ & 3,1765 & .09 \\
\hline $\begin{array}{l}\text { Haupteffekte } \\
\text { - Studiengang (Lehramt vs. Nicht-Lehramt) } \\
\text { - Fachgruppe (MINT- vs. kein-MINT-Fach) }\end{array}$ & $\begin{array}{l}12.67^{* *} \\
15.21^{* *}\end{array}$ & $\begin{array}{l}1,1517 \\
1,1517\end{array}$ & $\begin{array}{l}.01 \\
.01\end{array}$ & $\begin{array}{l}62.84^{* *} \\
59.62^{* *}\end{array}$ & $\begin{array}{l}1,1765 \\
1,1765\end{array}$ & $\begin{array}{l}.03 \\
.03\end{array}$ \\
\hline $\begin{array}{l}\text { Interaktion } \\
- \text { Studiengang } \times \text { Fachgruppe }\end{array}$ & 0.42 & 1,1517 & .00 & 0.53 & 1,1765 & .00 \\
\hline
\end{tabular}

Anmerkung: ${ }^{*} p<.05,{ }^{* *} p<.01,{ }^{* * *} p<.001$.

Tab. 3: Mittelwerte $(M)$, Standardabweichungen $(S D)$ und Standardfehler $(S E)$ der ICT Literacy von angehenden und fortgeschrittenen Studierenden getrennt nach Studiengruppe und Fächerkombination

\begin{tabular}{|c|c|c|c|c|c|c|c|c|}
\hline & \multirow[b]{2}{*}{ Studiengang } & \multirow[b]{2}{*}{ Fachgruppe } & \multicolumn{3}{|c|}{$\begin{array}{c}\text { Angehende } \\
\left.\text { Studierende ( } \mathrm{SC}_{4}\right)\end{array}$} & \multicolumn{3}{|c|}{$\begin{array}{l}\text { Fortgeschrittene } \\
\text { Studierende }\left(\mathrm{SC}_{5}\right)\end{array}$} \\
\hline & & & $M$ & $S D$ & $S E$ & $M$ & $S D$ & $S E$ \\
\hline$V_{1}:$ & $\begin{array}{l}\text { Lehramt } \\
\text { Nicht Lehramt }\end{array}$ & $\begin{array}{l}\text { Alle } \\
\text { Alle }\end{array}$ & $\begin{array}{l}381.83_{a} \\
412.21_{b}\end{array}$ & $\begin{array}{l}87.22 \\
95.81\end{array}$ & $\begin{array}{l}7.13 \\
2.58\end{array}$ & $\begin{array}{l}476.22{ }_{a} \\
522.74_{b}\end{array}$ & $\begin{array}{r}76.34 \\
109.19\end{array}$ & $\begin{array}{l}4.41 \\
2.68\end{array}$ \\
\hline V2: & Lehramt & $\begin{array}{l}\text { MINT-Fach } \\
\text { Kein MINT-Fach }\end{array}$ & $\begin{array}{l}399.88 \\
374.11\end{array}$ & $\begin{array}{l}80.97 \\
89.06\end{array}$ & $\begin{array}{r}11.86 \\
7.90\end{array}$ & $\begin{array}{l}501.43_{a} \\
463.93_{b}\end{array}$ & $\begin{array}{l}77.91 \\
73.56\end{array}$ & $\begin{array}{l}7.20 \\
5.11\end{array}$ \\
\hline$V_{3}:$ & Nicht Lehramt & $\begin{array}{l}\text { MINT-Fach } \\
\text { Kein MINT-Fach }\end{array}$ & $\begin{array}{l}428.54 a \\
398.06_{b}\end{array}$ & $\begin{array}{l}94.95 \\
94.29\end{array}$ & $\begin{array}{l}3.78 \\
3.53\end{array}$ & $\begin{array}{l}543.81_{a} \\
504.83_{b}\end{array}$ & $\begin{array}{l}108.25 \\
105.89\end{array}$ & $\begin{array}{l}3.89 \\
3.70\end{array}$ \\
\hline \multicolumn{3}{|c|}{ Gesamt } & 408.08 & 95.17 & 3.79 & 509.74 & 98.87 & 2.58 \\
\hline
\end{tabular}

Anmerkung: V1 = Vergleich 1, V2 = Vergleich 2, V3=Vergleich 3; Mittelwerte mit unterschiedlichen Subskripts unterscheiden sich in den Post-Hoc-Tests der ANOVA signifikant voneinander. 
großen Standardfehler ( $S E=11.86$ bzw. 7.90), die aus den vergleichsweise geringen Fallzahlen ( $n=71$ bzw. $n=150$; siehe Tab. 1 ) resultieren, nicht signifikant, sodass $\mathrm{H} 1 \mathrm{c}$ nicht gestützt wird.

\section{Entwicklung der ICT Literacy bis zum Ende des ersten Studienabschnitts (Fragestellung 2)}

Tabelle 4 zeigt die Resultate der dreifaktoriellen Varianzanalyse mit den Faktoren Studiengang, Fachgruppe und Startkohorte. Alle berücksichtigten Kontrollvariablen tragen signifikant zur Varianzaufklärung bei $\left(.003 \leq \mathrm{Eta}^{2} \leq .094\right)$. Die Haupteffekte weisen - neben den bereits beschriebenen Haupteffekten zum Studiengang und zur Fachgruppe - auf einen deutlichen $\mathrm{Zu}$ wachs in der ICT Literacy zwischen Studienbeginn und Ende des ersten Studienabschnitts hin. Der geschätzte Kompetenzzuwachs beträgt 102 Punkte (SC4: 408 Punkte, SC5: 510 Punkte; siehe Tab. 3), der unter Einbezug der Kontrollvariablen einem mittleren Effekt entspricht $\left(\mathrm{Eta}^{2}=.114\right.$; siehe Tab. 4).

Die Analyse der Interaktionseffekte weist erwartungskonform auf eine Zunahme der Kompetenzrückstände von Lehramtsstudierenden im Vergleich zu Studierenden anderer Fachrich- tungen hin (Interaktion Studiengang $\times$ Kohorte: $F_{1,3273}=5.47, p<.05 ; \mathrm{H} 2 \mathrm{a}$ ), wenngleich der Effekt sehr gering ausfällt $\left(\mathrm{Eta}^{2}=.001\right)$. Während der Unterschied bei den Lehramtsstudierenden 94 Punkte (382 vs. 476 Punkte) ausmacht, beträgt die Differenz bei den Studierenden anderer Fachrichtungen 111 Punkte (412 vs. 523 Punkte; siehe Tab. 3). Alle anderen Interaktionen fallen nicht signifikant aus, sodass Hypothese $2 \mathrm{~b}$, wonach Lehramtsstudierende ohne MINT-Fach besonders geringe Kompetenzzuwächse erzielen, zurückzuweisen ist.

\section{Kompetenzniveaus der Lehramts- studierenden (Fragestellung 3)}

In Bezug auf die kriterial verankerten Kompetenzniveaus zeigt sich korrespondierend mit den Ergebnissen der vorangegangenen Analysen, dass sowohl angehende als auch fortgeschrittene Lehramtsstudierende zu überproportional hohen Anteilen die jeweiligen Mindeststandards nicht erreichen (Tab. 5). Hypothesenkonform unterschreitet knapp ein Viertel der angehenden Lehramtsstudierenden (24,7\%) den festgelegten Mindeststandard, d.h. das Kompetenzniveau $\operatorname{Basic}(\mathrm{H} 3 \mathrm{a})$. Bei den Studierenden anderer Fachrichtungen fällt der Anteil mit 16,5\% deutlich geringer aus. Diese Verteilungsunterschiede

Tab. 4: Ergebnisse der dreifaktoriellen Varianzanalysen mit den Faktoren Studiengang, Fachgruppe und Kohorte (unter Kontrolle von Geschlecht, allgemeiner kognitiver Fähigkeit und elterlichem Bildungsniveau)

\begin{tabular}{|c|c|c|c|c|}
\hline Quelle & $\boldsymbol{F}$ & $d f$ & $p$ & Eta $^{2}$ \\
\hline Korrigiertes Modell & 191.27 & 10,3273 & $<.01$ & .370 \\
\hline $\begin{array}{l}\text { Haupteffekte } \\
\text { - Studiengang (Lehramt vs. kein Lehramt) } \\
\text { - Fachgruppe (MINT- vs. kein MINT-Fach) } \\
\text { - Kohorte (SC } 4 \text { vs. SC } 5 \text { ) }\end{array}$ & $\begin{array}{r}20.88 \\
16.19 \\
442.55\end{array}$ & $\begin{array}{l}1,3273 \\
1,3273 \\
1,3273\end{array}$ & $\begin{array}{l}<.01 \\
<.01 \\
<.01\end{array}$ & $\begin{array}{l}.006 \\
.004 \\
.114\end{array}$ \\
\hline $\begin{array}{l}\text { Interaktionen } \\
\text { - Studiengang } \times \text { Fachgruppe } \\
\text { - Studiengang } \times \text { Kohorte } \\
\text { - Fachgruppe } \times \text { Kohorte } \\
\text { - Studiengang } \times \text { Fachgruppe } \times \text { Kohorte }\end{array}$ & $\begin{array}{l}1.35 \\
5.47 \\
0.92 \\
0.22\end{array}$ & $\begin{array}{l}1,3273 \\
1,3273 \\
1,3273 \\
1,3273\end{array}$ & $\begin{array}{l}.245 \\
.019 \\
.337 \\
.639\end{array}$ & $\begin{array}{l}.001 \\
.001 \\
.000 \\
.000\end{array}$ \\
\hline $\begin{array}{l}\text { Kovariate } \\
\text { - Allgemeine kognitive Fähigkeit } \\
\text { - Geschlecht } \\
\text { - Elterliches Bildungsniveau }\end{array}$ & $\begin{array}{r}275.32 \\
221.89 \\
11.91\end{array}$ & $\begin{array}{l}1,3273 \\
1,3273 \\
1,3273\end{array}$ & $\begin{array}{c}<.01 \\
<.01 \\
.001\end{array}$ & $\begin{array}{l}.094 \\
.067 \\
.003\end{array}$ \\
\hline
\end{tabular}


14 Martin Senkbeil, Jan Marten Ihme, Christian Schöber

Tab. 5: Verteilung der angehenden und fortgeschrittenen Studierenden nach Studiengang auf die Kompetenzniveaus

\begin{tabular}{|l|l|c|c|c|c|} 
& \multicolumn{4}{|c|}{ Kompetenzniveau } \\
\hline Kohorte & Studiengang & N (absolut) & Below Basic & Basic & Proficient \\
\hline \multirow{2}{*}{ Angehende } & Lehramt & 207 & 24.7 & 66.3 & 9.0 \\
Studierende & Nicht-Lehramt & 1312 & 16.5 & 67.1 & 16.5 \\
& Gesamt & 1519 & 17.6 & 67.0 & 15.4 \\
\hline \multirow{2}{*}{$\begin{array}{l}\text { Fortgeschrittene } \\
\text { Studierende }\end{array}$} & Lehramt & 493 & 4.1 & 58.6 & 37.3 \\
\cline { 2 - 6 } & Nicht-Lehramt & 1273 & 1.7 & 41.2 & 57.1 \\
\hline
\end{tabular}

Anmerkung: Wertangaben bei den Kompetenzniveaus in Prozenten (gewichtet).

lassen sich statistisch absichern $\left(\chi^{2}[2]=14.24\right.$, $p<.01$ ), wobei der Cramer-V-Index von .10 auf einen kleinen Effekt (Cohen, 1988) hinweist.

Im sechsten Fachsemester erreicht nur etwas mehr als ein Drittel (37,3\%) der Lehramtsstudierenden das Kompetenzniveau Proficient. Trotz erheblicher Kompetenzzuwächse in der ersten Studienphase (94 Punkte) unterschreiten demnach knapp zwei Drittel (62,7\%) der fortgeschrittenen Lehramtsstudierenden den normativ festgelegten Standard, was deutlich über dem vorab vermuteten Prozentsatz liegt (H3b). Wiederum fällt der Anteil der Studierenden anderer Fachrichtungen, der den Mindeststandard (Kompetenzniveau Proficient) erreicht, mit $57,1 \%$ deutlich höher aus. Entsprechend lassen sich auch für die fortgeschrittenen Studierenden Verteilungsunterschiede in Abhängigkeit vom Studiengang statistisch absichern $\left(\chi^{2}[2]=60.39, p<.01\right)$. Der Cramer-V-Index von .19 weist wiederum auf einen vergleichsweisen kleinen Effekt hin, der jedoch deutlich ausgeprägter ist als zu Studienbeginn.

\section{Diskussion}

Mit dem vorliegenden Beitrag präsentieren wir Befunde zur Fragestellung, über welche digitale Kompetenzen Lehramtsstudierende zu verschiedenen Studienabschnitten verfügen. Da es hierzu bislang an belastbaren und verallgemeinerbaren Befunden fehlte (Herzig \& Martin, 2018; Lorenz et al., 2016), gingen wir die- ser Frage anhand einer Sekundäranalyse des Nationalen Bildungspanels nach. Angesichts der zunehmenden Bedeutung, die der schulischen Vermittlung digitaler Kompetenzen zukommt (BMBF, 2016; KMK, 2016), wurde zusätzlich untersucht, inwieweit sie vor Studienbeginn und gegen Ende des ersten Studienabschnitts über eine ausreichende ICT Literacy im Sinne kriterial festgelegter Mindeststandards verfügen.

Da das in dieser Arbeit untersuchte Konstrukt ICT Literacy eine hohe Affinität zu Konzepten der Medienkompetenz aufweist, kann ICT Literacy als Teilkonstrukt einer umfassenden Medienkompetenz aufgefasst werden, die wiederum eine notwendige Voraussetzung für den Erwerb medienpädagogischer Kompetenzen darstellt (Herzig \& Martin, 2017, 2018; Tulodziecki, 2012). Die anhand der NEPS-Daten ermittelten Ergebnisse sind demnach nicht nur als Beitrag zum Bildungsmonitoring zu verstehen, sondern haben auch Relevanz im Hinblick auf die empirische Erfassung medienpädagogischer Kompetenzen.

Im Folgenden werden die von den Lehramtsstudierenden erzielten digitalen Kompetenzen zunächst im Vergleich zu Studierenden anderer Fachrichtungen betrachtet. Anschließend werden sie im Hinblick auf zukünftige, berufliche Anforderungen, d.h. die Vermittlung digitaler Kompetenzen im schulischen Kontext, diskutiert. Wir enden mit Hinweisen auf Limitationen der vorliegenden Studie und damit verbundenen offenen Fragestellungen. 


\section{Einordnung der Befunde im Vergleich mit Studierenden anderer Fachrichtungen}

Die hier vorliegenden Ergebnisse erweitern die bisherige Befundlage zu digitalen Kompetenzen von Lehramtsstudierenden in Deutschland erheblich, da erstmalig Kennwerte anhand objektiv erhobener Testdaten an einer repräsentativen Stichprobe vorliegen. Dabei zeigte sich zunächst erwartungskonform, dass Lehramtsstudierende zu Studienbeginn und gegen Ende der ersten Ausbildungsphase im Sinne einer Negativ-Selektion eine geringere ICT Literacy aufweisen als Studierende anderer Fachrichtungen, wenngleich die Effektstärken nahezu durchgehend einem kleinen Effekt entsprechen. Entgegen der Erwartung konnte jedoch nur für fortgeschrittene Lehramtsstudierende ohne MINT-Fach ein negativer Binnenselektionseffekt im Vergleich zu Lehramtsstudierenden mit mindestens einem MINT-Fach ermittelt werden $(d=0.49)$. Für angehende Lehramtsstudierende ließ sich der Kompetenzrückstand aufgrund geringer Fallzahlen und der daraus resultierenden hohen Standardfehler nicht statistisch absichern, wie anhand einer Power-Analyse ermittelt werden konnte. Für Studierende anderer Fachrichtungen ohne MINT-Fach zeigten sich die vermuteten Kompetenznachteile zu beiden Zeitpunkten (0.32 $\leq d \leq 0.36)$. Diese Ergebnisse korrespondieren mit Befunden früherer Arbeiten, wonach Studierende technologiebezogener und mathematisch-naturwissenschaftlicher Studienfächer eine größere Affinität zu digitalen Medien aufweisen als Studierende der Sprach- und Kulturwissenschaften (Kleimann, Özkilic \& Göcks, 2008; Persike \& Friedrich, 2016).

Da der zu Studienbeginn ermittelte Kompetenzrückstand der angehenden Lehramtsstudierenden vergleichsweise gering ausfällt $(d=0.32)$, sollte dieser im Verlauf des Studiums - bei entsprechender Förderung digitaler Kompetenzen - prinzipiell kompensierbar sein. Die Ergebnisse aus der ersten Ausbildungsphase weisen jedoch auf eine leichte Zunahme des Kompe- tenzrückstands der Lehramtsstudierenden hin $(d=0.47)$, welcher sich auch statistisch absichern lässt. Auch wenn dieser Schereneffekt sehr klein ausfällt $\left(\mathrm{Eta}^{2}=.001\right)$, lassen sich weder für die Gesamtgruppe der Lehramtsstudierenden noch für Subgruppen (z. B. Lehramtsstudierende mit Wahl mindestens eines MINTFachs) Anzeichen für eine Verringerung der Kompetenzdefizite erkennen. Dieses Ergebnis korrespondiert mit seit längerer Zeit geäußerten Vermutungen und Hinweisen, wonach Lehramtsstudierende bereits zu Studienbeginn über vergleichsweise geringe digitale Kompetenzen verfügen und diese Kompetenzrückstände während des Studienverlaufs nicht kompensieren können (Herzig \& Grafe, 2007; Zylka \& Müller, 2011). Sehr anschaulich treten die Defizite der Lehramtsstudierenden bei Betrachtung der kriterial verankerten Kompetenzniveaus zutage. Sie manifestieren sich in überproportional hohen Anteilen von Lehramtsstudierenden, welche die jeweils erforderlichen Standards (angehende Studierende: Basic; fortgeschrittene Studierende: Proficient) nicht erreichen. Auch bei dieser Betrachtung deuten die Verteilungsunterschiede zwischen Lehramtsstudierenden und Studierenden anderer Fachrichtungen auf einen Schereneffekt hin.

\section{Einordnung der Befunde in Bezug auf zukünftige berufliche Anforderungen}

Da digitale Kompetenzen im Verlauf der universitären Ausbildungsphase vermittelt werden sollten (Herzig et al., 2015), stellt sich die Frage, was die Befunde für die weitere berufliche Entwicklung der angehenden Lehrerinnen und Lehrer, d.h. im Hinblick auf die Vermittlung digitaler Kompetenzen im schulischen Kontext, bedeuten. Dabei zeigt sich erstens, dass trotz erheblicher Kompetenzzuwächse im Verlauf der universitären Lehrerbildung nur etwas mehr als ein Drittel der Lehramtsstudierenden am Ende der ersten Studienphase den normativ festgelegten Mindeststandard (Kompetenzniveau Proficient: $37,3 \%$ ) erreicht. Es ist daher zu bezweifeln, dass alle angehenden Lehrkräfte der von 
der Bildungspolitik formulierten Anforderung gerecht werden, wonach alle Lehrkräfte selbst über allgemeine Medienkompetenz verfügen müssen und in ihren fachlichen Zuständigkeiten zugleich „Medienexperten“"werden (KMK, 2016, S. 23-24). Da gerade diese Studierenden zukünftig die Auseinandersetzung von Kindern und Jugendlichen mit digitalen Medien in didaktischer und erzieherischer Hinsicht anregen und unterstützen sollen, stehen diese Befunde im Widerspruch zu ihrer künftigen Rolle als Vermittlerinnen und Vermittler moderner Kulturtechniken (vgl. Herzig \& Grafe, 2007). Da ohne ausreichende Medienkompetenz der Erwerb medienpädagogischer Kompetenzen nicht gelingen kann, erscheint es für den Großteil der hier untersuchten Jahrgänge von Lehramtsstudierenden unwahrscheinlich, dass sie zukünftig in der Lage sind, digitale Medien zielgerichtet und kompetenzorientiert in unterrichtliche Lehr-Lernprozesse einzubeziehen (Zylka \& Müller, 2011).

Zweitens spiegeln die in dieser Arbeit ermittelten Befunde ganz offensichtlich den vielfach beschriebenen Befund wider, wonach es bisher in Deutschland weder flächendeckend umgesetzte Konzepte zur Förderung der Medienkompetenz angehender Lehrkräfte gab noch ihre didaktischen und methodischen Fähigkeiten zur Unterstützung des Medienkompetenzerwerbs von Schülerinnen und Schülern systematisch gefördert wurden. Es fehlt somit an einer flächendeckenden, verpflichtenden und prüfungsrelevanten Verankerung in pädagogischen Studien- und Ausbildungseinrichtungen, die eine angemessene „Grundbildung Medien“ für angehende Lehrpersonen ermöglichen (zusammenfassend: Eickelmann et al., 2016). Vor diesem Hintergrund ist es wenig überraschend, dass sich zu Studienbeginn bestehende Kompetenzrückstände in der ICT Literacy während der universitären Ausbildung manifestieren oder sogar noch vergrößern. Diese Ergebnisse unterstreichen somit die Dringlichkeit einer zukunftsfähigen Veränderung der Lehrerausbildung, wie sie beispielsweise die KMK (2016) einfordert. Entsprechend zeigt eine neuere Un- tersuchung, dass sich Lehrkräfte mehrheitlich (mehr als $80 \%$ ) sowohl in der universitären Lehrerausbildung als auch in der Referendarausbildung eine bessere Vorbereitung auf den Einsatz digitaler Medien im Unterricht wünschen (Eickelmann et al., 2016).

Insgesamt deuten die Ergebnisse auf ein grundlegendes Problem der digitalen Bildung in Deutschland hin, welches bereits in der internationalen Schulleistungsstudie ICILS 2013 zutage trat. Dort zeigte sich unter anderem, dass nur knapp die Hälfte der Gymnasiastinnen und Gymnasiasten (48\%), aus denen sich die zukünftigen (Lehramts-) Studierenden rekrutieren, ein Kompetenzniveau erreicht, welches eine selbstbestimmte und reflektierte Teilhabe in einer digitalisierten Welt erwarten lässt (z. B. selbstständiges Lösen informationsbezogener Problemstellungen; Bos et al., 2014; Eickelmann, 2017). Auch wenn die Studien NEPS und ICILS 2013 methodisch und in ihrer Anlage nicht direkt miteinander vergleichbar sind, scheinen sich die in Klasse 8 ermittelten Kompetenzdefizite zahlreicher Schülerinnen und Schüler bis ins Studium fortzusetzen, d. h. während der weiteren Schullaufbahn nicht mehr vollständig kompensiert zu werden.

\section{Limitationen}

Mit der vorgestellten Studie konnten zwar neue Einblicke über die ICT Literacy von Lehramtsstudierenden generiert werden, jedoch sind ebenfalls einschränkende Aspekte der Untersuchung zu berücksichtigen. Zum ersten wurde die ICT Literacy der angehenden Studierenden ein Jahr vor Studienbeginn, d. h. in Klasse 12 erhoben. Veränderungen in der ICT Literacy zwischen Messzeitpunkt und Aufnahme des Studiums sind daher nicht gänzlich auszuschließen, wenngleich diese aufgrund der unsystematischen und vergleichsweise geringen Nutzung digitaler Medien in der Schule nicht substanziell sein sollten (Eickelmann et al., 2017). Nichtsdestotrotz wäre eine Kompetenzmessung unmittelbar vor Studienbeginn vorteilhafter. 
Zum zweiten wurde keine wirkliche Längsschnittsanalyse durchgeführt, sondern die Entwicklung der ICT Literacy anhand zweier Querschnittsstichproben geschätzt. Trotz der Kontrolle zentraler Eingangsvoraussetzungen (z. B. allgemeine kognitive Fähigkeit) und nahezu identischer Messzeitpunkte bleiben beispielsweise Kohorteneffekte unberücksichtigt. Es bedarf demnach realer Längsschnittstudien, um Kompetenzzuwächse angemessener zu modellieren. Dies gilt ebenso zur Klärung der Frage, inwieweit das schlechte Abschneiden der angehenden Lehramtsstudierenden auf eine vergleichsweise große Distanz zu digitalen Medien (medialer Habitus) zurückzuführen ist. Aufgrund der vorliegenden Daten ist diese Frage nicht zu beantworten.

Zum dritten stellt die in dieser Studie untersuchte ICT Literacy nur ein Teilkonstrukt einer umfassenderen Medienkompetenz dar und erfasst Fertigkeiten und Fähigkeiten im Sinne einer digitalen Grundbildung, die von allen Studierenden erfüllt sein sollten (vgl. Senkbeil et al., 2019), aber keine methodischen oder didaktischen Kompetenzen im Hinblick auf LehrLernprozesse mit digitalen Medien im Unterricht (Herzig \& Martin, 2018). Daher sind auch weiterhin unzureichende Kenntnisse über die medienpädagogischen Kompetenzen angehender wie auch aktiver Lehrkräfte zu konstatieren. Da aber mittlerweile erste Instrumente $\mathrm{zu}$ ihrer Messung entwickelt wurden (Herzig et al., 2015), könnten in absehbarer Zeit auch hierzu erste, empirisch abgesicherte Erkenntnisse vorliegen.

\section{Literatur}

Barak, M. (2014). Closing the gap between attitudes and perceptions about ICT-enhanced learning among preservice STEM teachers. Journal of Science Education and Technology, 23, 1-14. https://doi.org/10.1007/s10956013-9446-8

Bertelsmann Stiftung, CHE Centrum für Hochschulentwicklung, Deutsche Telekom Stiftung \& Stifterverband für die Deutsche Wissenschaft (Hrsg.). (2018). Lehramtsstudium in der digitalen Welt - Professionelle Vorbereitung auf den Unterricht mit digitalen Medien?! Eine Sonderpublikation aus dem Projekt Monitor Lehrerbildung. Zugriff am 1. 11.0218. Verfügbar unter: https:// www.monitor-lehrerbildung.de/export/sites/default/.
content/Downloads/Broschuere_Lehrerbildung-inder-digitalen-Welt.pdf.

Beutenagel, B., Dittmann, C., Meyer, R. \& Baumhauer, M. (2018). Berufsbegleitende Studiengänge im MINT-Sektor. Düsseldorf: Hans-Böckler-Stiftung.

Blossfeld, H.-P., Roßbach, H.-G. \& von Maurice, J. (Eds.) (2011). Education as a lifelong process. The German National Educational Panel Study (NEPS). Zeitschrift für Erziehungswissenschaft, Special Issue 14. https://doi. org/10.1007/s11618-011-0179-2

$\mathrm{BMBF}$ [Bundesministerium für Bildung und Forschung] (2016). Bildungsoffensive für die digitale Wissensgesellschaft. Berlin: BMBF.

Bos, W., Eickelmann, B., Gerick, J., Goldhammer, F., Schaumburg, H., Schwippert, K., ... Wendt, H. (Hrsg.) (2014). ICILS 2013 - Computer-und informationsbezogene Kompetenzen von Schülerinnen und Schülern in der 8. Jahrgangsstufe im internationalen Vergleich. Münster: Waxmann.

Brauns, H., Scherer, S. \& Steinmann, S. (2003), The CASMIN educational classification in international comparative research. In J. H. P. Hoffmeyer-Zlotnik \& C. Wolf (Hrsg.), Advances in cross-national comparison. An European working book for demographic and socio-economic variables (S. 196-211). Amsterdam. https://doi. org/10.1007/978-1-4419-9186-7_11

Cohen, J. (1988). Statistical Power Analysis for the Behavioral Sciences. Hillsdale: Lawrence Erlbaum Associates.

Eickelmann, B. (2017). Schulische Medienkompetenzförderung. In H. Gapsaki, M. Oberle \& W. Staufer (Hrsg.), Medienkompetenz. Herausforderung für Politik, politische Bildung und Medienbildung (S. 146-154). Bonn: Bundeszentrale für Politische Bildung.

Eickelmann, B., Lorenz, R. \& Endberg, M. (2016). Die eingeschätzte Relevanz der Phasen der Lehrerausbildung hinsichtlich der Vermittlung didaktischer und methodischer Kompetenzen von Lehrpersonen für den schulischen Einsatz digitaler Medien in Deutschland und im Bundesländervergleich. In W. Bos, R. Lorenz, M. Endberg, B. Eickelmann, R. Kammerl \& S. Welling (Hrsg.), Schule digital-der Länderindikator 2016. Kompetenzen von Lehrpersonen der Sekundarstufe I im Umgang mit digitalen Medien im Bundesländervergleich (S. 149-182). Münster: Waxmann.

Eickelmann, B., Lorenz, R. \& Endberg, M. (2017). Lernaktivitäten mit digitalen Medien im Fachunterricht der Sekundarstufe I im Bundesländervergleich mit besonderem Fokus auf MINT-Fächer. In R. Lorenz, W. Bos, M. Endberg, B. Eickelmann, S. Grafe \& J. Vahrenhold (Hrsg.), Schule digital - der Länderindikator 2017. Schulische Medienbildung in der Sekundarstufe I mit besonderem Fokus auf MINT-Fächer im Bundesländervergleich und Trends von 2015 bis 2017 (S. 231-260). Münster: Waxmann.

Fraillon, J., Schulz, W. \& Ainley, J. (2013). International Computer and Information Literacy Study: Assessment framework. Amsterdam: IEA. https://doi.org/10.1007/ 978-3-319-14222-7_4

Haberkorn, K. \& Pohl, $\bar{S}$. (2013). Cognitive basic skills Data in the scientific use file. Bamberg: University of Bamberg.

Hatlevik, O.E. (2017). Examining the relationship between teachers' self-efficacy, their digital competence, strategies to evaluate information, and use of ICT at school. Scandinavian Journal of Educational Reaserch, 61, 555567. https://doi.org/10.1080/00313831.2016.11725 01 
Hermida, M., Hielscher, M. \& Petko, D. (2017). Medienkompetenz messen: Die Entwicklung des MedienprofiTests in der Schweiz. Medienpädagogik, 1, 38-60.

Herzig, N. (2014). Medien in der Schule. In A. Tillmann, S. Fleicher \& K.-U. Hugger (Hrsg.), Handbuch Kinder und Medien (S. 531-546). Wiesbaden: Springer VS. https://doi.org/10.1007/978-3-531-18997-0 40

Herzig, B. \& Grafe, S. (2007). Digitale Medien in der Schule. Standortbestimmung und Handlungsempfehlungen fir die Zukunft. Bonn: Deutsche Telekom AG.

Herzig, B. \& Martin, A. (2017). Erfassung und Messbarkeit von Medienkompetenz als wichtige Voraussetzung für politische Bildung. In H. Gapski, M. Oberle \& W. Staufer (Hrsg.), Medienkompetenz. Herausforderung für Politik, politische Bildung und Medienbildung (S. 126135). Bonn: Bundeszentrale für Politische Bildung.

Herzig, B. \& Martin, A. (2018). Lehrerbildung in der digitalen Welt. In S. Ladel, J. Knopf \& A. Weinberger (Hrsg.), Digitalisierung und Bildung (S. 89-113) Wiesbaden: Springer VS. https://doi.org/10.1007/9783-658-18333-2_6

Herzig, B., Martin, A., Schaper, N. \& Ossenschmidt, D (2015). Modellierung und Messung medienpädagogischer Kompetenz - Grundlagen und erste Ergebnisse. In B. Koch-Priewe, A. Köker, J. Seifried \& E. Wuttke (Hrsg.), Kompetenzerwerb an Hochschulen: Modellierung und Messung. Bad Heilbronn: Klinkhardt.

Kammerl, R. \& Mayrberger, K. (2011). Medienpädagogik in der Lehrerinnen- und Lehrerbildung in Deutschland: Aktuelle Situation und Desiderata. Beiträge zur Lehrerinnen- und Lehrerbildung, 29, 172-184.

Kammerl, R. \& Pannarale, S. (2007). Students in higher education and teacher training programs in Germany: Their internet use, media literacy, and attitude toward elearning. In R. Carlsen, K. McFerrin, J. Price, R. Weber \& D. A. Willis (Eds.), Proceedings of the Society for Information Technology and Teacher Education International Conference 2007 (S. 3067-3072). Chesapeake, VA: AACE.

Kleimann, B., Özkilic, M. \& Göcks, M. (2008). Studieren im Web 2.0. Studienbezogene Web- und E-LearningDienste. Zugriff am 21.2.2019 unter https://hisbus.his. de/hisbus/docs/hisbus21.pdf. Zugegriffen: 11.9.2018.

Klieme, E., Hartig, J. \& Rauch, D. (2008). The concept of competence in educational contexts. In J. Hartig, E. Klieme \& D. Leutner (Hrsg.), Assessment of competencies in educational contexts (S. 3-22). Göttingen: Hogrefe.

Klusmann, U., Trautwein, U., Lüdtke, O., Kunter, M. \& Baumert, J. (2009). Eingangsvoraussetzungen beim Studienbeginn. Werden die Lehramtskandidaten unterschätzt? Zeitschrift für Pädagogische Psychologie, 23 265-278. https://doi.org/10.1024/1010-0652.23.34. 265

KMK [Kultusministerkonferenz] (2016). Bildung in der digitalen Welt. Strategie der Kultusministerkonferenz. Bonn: KMK.

Kommer, S. \& Biermann, R. (2012). Der mediale Habitus von (angehenden) LehrerInnen. Medienbezogene Dispositionen und Medienhandeln von Lehramtsstudierenden. In R. Schulz-Zander, B. Eickelmann, H. Moser, H. Niesyto \& P. Grell (Hrsg.), Jahrbuch Medienpädagogik 9 (S. 8-108). Wiesbaden: Springer VS. https://doi.org/10.1007/978-3-531-94219-3 5

Kuhlemeier, H. \& Hemker, B. (2007). The impact of computer use at home on students' internet skills. Computers \& Education, 49, 460-480. https://doi.org/10.10 16/j.compedu.2005.10.004
Lorenz, R., Gerick, J., Wendt, H. \& Weischenberg, J. (2016). Einschätzung von Sekundarstufenlehrkräften zu ihren Kompetenzen im Umgang mit neuen Technologien in Lehr- und Lernprozessen. In B. Eickelmann, J. Gerick, K. Drossel \& W. Bos (Hrsg.), ICILS 2013. Vertiefende Analysen zu computer-und informationsbezogenen Kompetenzen von Jugendlichen (S. 119-142). Münster: Waxmann.

Pant, H. A., Tiffin-Richards, S. P. \& Köller, O. (2010). Standard-Setting für Kompetenztests im Large-Scale-Assessment. Zeitschrift für Pädagogik, 56. Beiheft, 175-188.

Persike, M. \& Friedrich, J. D. (2016). Lernen mit digitalen Medien aus Studierendenperspektive. Arbeitspapier Nr. 17. Berlin: Hochschulforum Digitalisierung. Zugriff am 21.2.2019 unter https://hochschulforumdigitalisierung.de/sites/default/files/dateien/HFD_AP_Nr_17 Lernen_mit_digitalen_Medien_aus_Studierendenperspektive.pdf

Pettersson, F. (2018). On the issues of digital competence in educational contexts - a review of the literature. Education and Information Technologies, 23, 1005-1021. https://doi.org/10.1007/s10639-017-9649-3

Pohl, S. \& Carstensen, C. H. (2013). Scaling of competence tests in the National Educational Panel Study - Many questions, some answers, and further challenges. Journal for Educational Research Online, 5, 189-216.

Reimer, D. \& Pollak, R. (2009). Educational expansion and its consequences for vertical and horizontal inequalities in access to higher education in west Germany. European Sociological Review, 26, 415-430. https://doi.org/ 10.1093/esr/jcp029

Rost, J. (2004). Lehrbuch Testtheorie - Testkonstruktion. Bern: Hans Huber.

Rubin, D. B. (1987). Multiple Imputation for Nonresponse in Surveys. J. Wiley \& Sons: New York. https://doi.org/ $10.1002 / 9780470316696$

Schiefner-Rohs, M. \& Hofhues, S. (2018). Zurück in die Zukunft. Anforderungen an Medienbildung in der Ausund Weiterbildung von Lehrpersonen am Beispiel eines Praxis- und Entwicklungsprojekts. Medienpädagogik, 31, 58-77.

Senkbeil, M., Ihme, J. M. \& Wittwer, J. (2013). Entwicklung und erste Validierung eines Tests zur Erfassung technologischer und informationsbezogener Literacy (TILT) für Jugendliche am Ende der Sekundarstufe I. Zeitschrift für Erziehungswissenschaft, 16, 671-691. https://doi.org/10.1007/s11618-013-0446-5

Senkbeil, M., Schöber, C. \& Ihme, J. M. (2019). Wie gut sind angehende und fortgeschrittene Studierende auf das Leben und Arbeiten in der digitalen Welt vorbereitet? Ergebnisse eines Standard Setting-Verfahrens zur Beschreibung von ICT-bezogenen Kompetenzniveaus. Zeitschrift für Erziehungswissenschaft, 22, 1359-1384. https://doi.org/10.1007/s11618-019-00914-z

Tulodziecki, G. (2010). Standards für die Medienbildung als eine Grundlage für die empirische Erfassung von Medienkompetenz-Niveaus. In B. Herzig, D. M. Meister, H. Moser \& H. Niesyto (Hrsg.), Jahrbuch Medienpädagogik. Medienkompetenz und Web 2.0 (S. 81-101). Wiesbaden: Springer VS. https://doi.org/10.1007/9783-531-92135-8_5

Tulodziecki, G. (2012). Medienpädagogische Kompetenz und Standards in der Lehrerbildung. In R. Schulz-Zander, B. Eickelmann, H. Moser, H. Niesyto \& P. Grell (Hrsg.), Jahrbuch Medienpädagogik 9 (S. 271-297). Wiesbaden:SpringerVS. https://doi.org/10.1007/9783-531-94219-3 13 
Tulodziecki, G. (2015). Dimensionen von Medienbildung. Ein konzeptioneller Rahmen für medienpädagogisches Handeln. Medienpädagogik, 6, 31-49.

Wu, M.L., Adams, R. J., Wilson, M.R. \& Haldane, S. (2007). ACER ConQuest 2.0-Generalised item response modeling software. Camberwell: ACER Press.

Zylka, J. \& Müller, W. (2011). Fundierung digitaler Medien im formalen Bildungswesen am Beispiel einer Fallstudie zu digitalen Medienkompetenzen. In T. Köhler \& J. Neumann (Hrsg.), Wissensgemeinschaften. Digitale Medien - Öffnung und Offenheit in Forschung und Lehre (S. 250-260). Münster: Waxmann.

\section{Martin Senkbeil \\ Jan Marten Ihme \\ Christian Schöber}

Leibniz-Institut für die Pädagogik

der Naturwissenschaften und Mathematik (IPN)

an der Universität Kiel

Olshausenstraße 62

D-24118 Kiel

E-Mail: senkbeil@ipn.uni-kiel.de ihme@ipn.uni-kiel.de schoeber@ipn.uni-kiel.de 